\title{
Nueva estrategia de diagnóstico para la sífilis primaria
}

Las pruebas serológicas para detectar la sífilis constituyen en la actualidad la base fundamental del diagnóstico de esta enfermedad en el mundo. Estas pruebas son poco costosas, simples y están disponibles ampliamente. Las pruebas llamadas no treponémicas detectan anticuerpos antilipídicos en el suero o el líquido cefalorraquídeo y pueden emplearse para cuantificar la respuesta de anticuerpos mediante la dilución seriada de las muestras. Sin embargo, estas pruebas - como la de laboratorio para la enfermedad venérea (VDRL) y la prueba rápida con reagina plasmática (RPR) — tienen baja especificidad y sensibilidad en los casos de sífilis primaria y en las etapas tardías de la infección. Por su parte, las pruebas treponémicas - como la que detecta Treponema pallidum mediante la aglutinación de partículas (TP-PA) - están dirigidas a anticuerpos específicos contra esta espiroqueta y son más sensibles debido a que los anticuerpos contra T. pallidum preceden por 
lo general a los anticuerpos antilipídicos. En general, la sensibilidad de la prueba TP-PA es mayor que la de VDRL y la de RPR ( $88 \%$ frente a $78 \%$ y $86 \%$, respectivamente).

Sin embargo, a pesar de que las pruebas treponémicas son más específicas que las no treponémicas, no se recomienda su uso en pacientes que hayan tenido esta infección con anterioridad, debido a la prolongada permanencia de los anticuerpos en el organismo, que hace que el resultado de esta prueba sea positivo por el resto de la vida. Por ello se recomienda utilizar estas pruebas para programas de tamizaje y como diagnóstico de primera línea.

En este trabajo se analizó mediante un estudio transversal la sensibilidad de las pruebas serológicas para el diagnóstico de la sífilis y cuál de estas pruebas es capaz de detectar más casos que el sistema actual basado en la realización de una prueba no treponémica (VDRL) y su confirmación mediante una prueba treponémica. Como diagnóstico de referencia se utilizó el examen por microscopía de campo oscuro (MCO).

La sensibilidad de la prueba TP-PA, utilizada como primera elección para el tamizaje, fue de $86 \%$ (intervalo de confianza de 95\% [IC95\%]: 78 a 92\%), aunque fue menor en personas seropositivas al $\mathrm{VIH}$ que en los seronegativos (55\% frente a $77 \%$; $P=0,05)$. La estrategia actual de confirmar los resultados positivos de las pruebas no treponémicas mediante pruebas treponémicas hubiera detectado solamente $71 \%$ de los casos (IC95\%: 61 a 79\%). Al parecer, esta estrategia resultó menos sensible porque depende de un resultado positivo en la prueba diagnóstica inicial (no treponémica), de menor sensibilidad en los casos de sífilis primaria, aunque se desconocen las bases biológicas de las diferencias observadas en el comportamiento de las diversas pruebas diagnósticas. La prueba de la RPR y la de VDRL tuvieron resultados similares ante los casos de sífilis primaria (71\%; IC95\%: 56 a 83\%).

A pesar de que en este estudio - como en muchos anteriores-, la sensibilidad de las pruebas treponémicas y la de las no treponémica fueron similares, la estrategia diagnóstica basada en el uso inicial de la prueba TP-AP seguida de la cuantificación del título de anticuerpos con un sistema no treponémico permitió aumentar el número de casos sospechados de sífilis primaria que se detectaron. Esta estrategia puede ser particularmente útil en los lugares donde no se dispone de condiciones para realizar la MCO.

Los cambios recomendados en la estrategia de diagnóstico implican adoptar una prueba treponémicas, como la TP-AP, como sistema diagnóstico de primera línea. Esta transición puede implementarse sin dificultades, ya que hay numerosos sistemas treponémicos disponibles, se usan con gran frecuencia y no requieren cambios en la tecnología empleada. El título de anticuerpos mediante sistemas no treponémicos debe realizarse siempre para evaluar la respuesta al tratamiento.

A pesar de que aún se requiere desarrollar pruebas diagnósticas más sensibles y específicas, la estrategia propuesta puede contribuir a mejorar el diagnóstico actual de la sífilis primaria. (Creegan $\mathrm{L}$, Bauer HM, Samuel M, Klausner J, Liska S, Bolan G. An evaluation of the relative sensitivities of the venereal disease research laboratory (VDRL) test and the Treponema pallidum particle agglutination (TPPA) test among patients diagnosed with primary syphilis. Sex Transm Dis. 2007;34(12):1016-8.) 